\title{
Usefulness of Microscan System panels with EUCAST clinical breakpoints to evaluate the antimicrobial susceptibility of ß-lactamase producing- Gram negative isolates
}

\author{
Elisabetta Nucleo', Melissa Spalla',Aurora Piazza', Roberta Migliavacca', Piero Micheletti², \\ Laura Pagani' \\ I Sezione di Microbiologia, Dip. S.M.E.C., Università degli Studi di Pavia \\ 2 Sezione di Anatomia Umana, Dip. Medicina Sperimentale, Università degli Studi di Pavia
}

Key words: Microscan System panels; Gram negative EUCAST clinical breakpoints; B-Lactamases

Utilizzo dei pannelli Microscan secondo i breakpoint EUCAST per la valutazione della sensibilità antimicrobica di isolati Gram-negativi beta-lattamasi-produttori

\section{SUMMARY}

The study aimed to evaluate the ability of NBC45, NBC46 and NB40 Microscan (MS) panels, updated to 2010 EUCAST breakpoints, to identify at species level and to correctly define the susceptibility to B-lactams of 6 I B-lactamases (BLs) producing Gram-negative isolates.

A collection of 73 fully identified strains was analyzed: 2 I Klebsiella spp., 17 E. coli, 15 P. mirabilis, 9 A. baumannii (Ab), 7 P. aeruginosa and 4 Enterobacter spp..

6I/73 were BLs and/or carbapenemases producers: 15 were CTX-M-I/-2/-I4/-I5 positive, and among them two were also VIM-I positive. Four were TEM-52/-92, 3 PER-I, 2 SHV-I2/-I8 and 6 CMY-I6 producers, while II were KPC-2/-3, 9 OXA-5I/-58/-23, 8 VIM-I and 2 IMP-I3 positive. One K. oxytoca K-I iper-producer, II non-BL producers/ATCC control strains and a OprD2 porin lacking $P$. aeruginosa were also included. All isolates were identified by Api-20E and VITEK-2 System and antibiotic susceptibilities were obtained by broth microdilution method. Resistance genes were identified by PCR and sequencing. All 73 isolates were correctly identified and a complete agreement for susceptibility patterns was observed for both ATCC control strains and non-BL clinical isolates. MS failed to detect a BL/Extended-Spectrum- $\beta$-Lactamase (ESâL) production in $5 / 6 \mathrm{I}$ cases: any ESBL alert was detected using NBC46 panel for 3/I5 CTX-M positive strains and 2 VIM-I/CTX-M-I5 producing $K$. pneumoniae isolates. Intermediate resistance to cefoxitin (MIC $16 \mathrm{mg} / \mathrm{L}$ ), susceptibility to cefepime (MIC $\leq \mathrm{I} \mathrm{mg/L}$ ) and to piperacillin/tazobactam (MIC $\leq 8 \mathrm{mg} / \mathrm{L}$ ) were correctly observed for CMY-16 producers.

The KPC producers were always correctly detected, with MIC values of $\mathrm{I}-\geq 8 \mathrm{mg} / \mathrm{L}$ for ertapenem (ETP), according to previously results. All VIM-I producers resulted intermediate/resistant to imipenem (IP) and meropenem (MP); decreased MIC values were observed in $2 / 8$ cases. Carbapenem MICs $>8 \mathrm{mg} / \mathrm{L}$ were detected for IP- 13 P. aeruginosa producers; 6/9 OXA carbapenemases- producing $A b$ showed IP MIC $\geq 8 \mathrm{mg} / \mathrm{L}$ and $3 / 6 \mathrm{MP}$ MIC $\geq 8 \mathrm{mg} / \mathrm{L}$.

$3 / 9$ Ab OXA-58/-5I producers, tested using NB40 panel, were intermediate or resistant to doripenem and meropenem. Regarding the detection of BLs overall agreement between MS and reference methods was $91.9 \%$. Carbapenems MIC values resulted a fold lower than previously determined.

Nevertheless using 2010 EUCAST breakpoints for ETP, MP and IP was possible to detect all carbapenemases- producers. MS System represents a useful tool to perform identification of BL- producing Gram negative bacteria.

\section{INTRODUZIONE}

La produzione di $\beta$-lattamasi a spettro esteso $(\mathrm{ES} \beta \mathrm{L})$ da parte dei batteri Gram-negativi rappresenta attualmente un problema di rilevante impatto clinico-terapeutico ed epidemiologico sia per le strutture ospedaliere per acuti, che per lungodegenze riabilitative e residenze sanitarie assistenziali (RSA).
La produzione di ES $\beta L$ determina resistenza verso cefalosporine a spettro allargato ed aztreonam, ma i microorganismi ES $\beta$ L- produttori mostrano frequentemente una resistenza estesa ad altre classi di antibiotici, quali gli aminoglicosidi ed i chinoloni.

Per la corretta refertazione della sensibilità agli antibiotici, il primo elemento critico consiste nel-

\section{Corresponding author: Elisabetta Nucleo}

Sez. Microbiologia, Dip. Scienze Morfologiche, Eidologiche e Cliniche - Università di Pavia

Viale Brambilla, 74 - 27I00 Pavia - Tel.: 0382984 I45 - Fax: 03825484255

E-mail: elisabetta.nucleo@unipv.it 
l'esatta identificazione dei batteri a livello di specie. I Laboratori di Microbiologia Clinica avvertono la necessità di utilizzare metodi di rilevamento che riflettano con accuratezza sempre maggiore i livelli di antibiotico-resistenza, permettendo di individuare quei meccanismi che possono influenzare l'outcome clinico.

Soprattutto nel caso del trattamento di infezioni gravi, qualsiasi ritardo nel riconoscere isolati multi-resistenti (MDR) può portare ad una scelta terapeutica non idonea sia in termini qualitativi che di dosaggio antibiotico.

Il rilevamento dei batteri ES $\beta \mathrm{L}$ e carbapenemasiproduttori risulta inoltre di fondamentale importanza ai fini di controllo delle infezioni e sorveglianza epidemiologica locale.

Lo scopo dello studio è stato valutare le performance dei pannelli NBC45, NBC46 ed NB40 Microscan (Siemens). E' stata determinata la capacità di identificare e segnalare correttamente isolati clinici Gram-negativi caratterizzati da meccanismi di resistenza noti e già investigati con tecniche fenotipiche e molecolari.

\section{MATERIALI E METODI}

Nel periodo Agosto 2010 - Novembre 2010 sono stati selezionati e processati presso il Laboratorio di Microbiologia del Dipartimento S. M. E. C. dell'Università di Pavia un totale di 73 isolati clinici. Tali isolati comprendevano: 21 ceppi di Klebsiella spp., 17 di Escherichia coli, 15 di Proteus mirabilis, 9 di Acinetobacter baumannii, 7 di Pseudomonas aeruginosa e 4 di Enterobacter spp.. 61/73 (83,5 \%) stipiti erano $\beta \mathrm{L}-$, ES $\beta \mathrm{L}$ - e/o carbapenemasi- produttori: $15 / 61$ isolati erano CTX-M-1/-2/-14/-15 produttori (2/15 di questi erano anche VIM-1 positivi), 4/61 producevano gli enzimi TEM-52/-92, 3/61 l'enzima PER-1, 2/61 gli enzimi SHV-12/-18, 6/61 la cefalosporinasi acquisita CMY-16, 11/61 le carbapenemasi KPC-2/-3, 9/61 gli enzimi OXA-51/-58/-23, 8/61 la Metallo- $\beta$-Lattamasi (MBL) VIM-1 e 2/61 la IMP-13 (Tabella 1).

Sono stati inclusi nello studio anche uno stipite di $K$. oxytoca $\mathrm{K}-1$ iper-produttore, 11 stipiti non- $\beta \mathrm{L}$ produttori ed uno stipite di $P$. aeruginosa mancante della porina OprD2.

Gli isolati clinici, raccolti presso Strutture Ospedaliere Italiane per acuti e lungodegenti site sull'intero territorio Nazionale, provenivano principalmente da materiali quali urine e sangue, ma anche da campioni respiratori, tamponi vari e feci. Tutti gli isolati sono stati identificati mediante il sistema Api-20E e VITEK-2, mentre il profilo di sensibilità è stato ottenuto mediante micro-diluizione in brodo (E-test limitatatamente ad alcuni antibiotici).

L'identificazione delle $\beta$ L è stata ottenuta median- te amplificazione utilizzando primer adatti al rilevamento dei geni blaTEM, blaSHV, blaCTX-M, blaPER, blaCMY, blaKPC, blaVIM, blaIMP e blaOXA e successivo sequenziamento.

Identificazione batterica, valutazione delle MIC e segnalazione del meccanismo di resistenza sono stati ottenuti mediante i pannelli Microscan NBC45, NBC46 e NB40; i risultati sono stati interpretati secondo le linee guida EUCAST 2010.

\section{RISULTATI}

Tutti i 73 isolati sono stati correttamente identificati con pannelli NBC 45/46 dal sistema Microscan (MS).

E'stato inoltre osservato un completo accordo, sia nel caso dei ceppi ATCC che per gli isolati clinici non BL- produttori, fra i valori di MIC ottenuti con MS e quelli determinati mediante micro-diluizione in brodo.

Per uno stipite di K. oxytoca K1- iper-produttore MS non ha segnalato la produzione di ES $\beta$ L ed ha correttamente evidenziato sensibilità nei confronti di amikacina, cefoxitina, ceftazidime, ETP, fosfomicina, gentamicina, IP, MP, tetraciclina, tigeciclina e tobramicina.

Resistenza verso i carbapenemi e sensibilità solo nei confronti di fosfomicina e colistina sono state correttamente evidenziate per uno stipite di $P$. aeruginosa carbapenemico-resistente a causa di un difetto di permeabilità.

MS ha fallito nel rilevare la produzione di ES $\beta \mathrm{L}$ in 5/61 casi: nessun Alert ES $\beta$ L è stato segnalato utilizzando il panello NBC46 per 3/15 ceppi CTX-M positivi e nel caso di due isolati di $K$. pneumoniae VIM-1/CTX-M-15 co-produttori.

Risulta comunque fondamentale sottolineare che $\mathrm{i}$ pannelli MS hanno in tutti e cinque i casi definito elevati valori di MIC ( $8 \mathrm{mg} / \mathrm{L}->32 \mathrm{mg} / \mathrm{L}$ ) per le cefalosporine di terza e quarta generazione (Tabella 2).

Per i due isolati di K. pneumoniae VIM-1/CTXM-15 produttori, i valori di MIC forniti da Microscan per IP (pari a $4 \mathrm{mg} / \mathrm{L}$ ) sono risultati sovrapponibili a quelli ottenuti mediante microdiluizione in brodo; le MIC per MP risultavano invece sottostimate per una diluizione.

I range di MIC ottenuti (per specie batterica) nel caso dei rimanenti isolati ES $\beta$ L-produttori sono riportati in tabella 2.

Per i sei ceppi di P. mirabilis CMY-16- produttori, il fenotipo di resistenza ottenuto con Microscan (pannello NBC 45) è risultato in linea con quello atteso: un valore di MIC per la cefoxitina (CFX) pari a $16 \mathrm{mg} / \mathrm{L}$, sensibilità alla piperacillina/tazobactam (MIC $<8 \mathrm{mg} / \mathrm{L}$ ) e valori di MIC del cefepime (CPE) compresi tra $0,5-8 \mathrm{mg} / \mathrm{L}$ sono stati correttamente osservati per tutti i ceppi studiati. 
Undici dei 61 ceppi in studio erano produttori di una carbapenemasi di tipo KPC-2/3.

MS ha consentito il corretto rilevamento di tutti gli isolati KPC produttori, con valori di MIC per l'ertapenem (ETP) superiori ad $1 \mathrm{mg} / \mathrm{L}$, in accordo con i risultati ottenuti precedentemente sia in brodo-macrodiluizione che con E-test.

Nel caso di isolati carbapenemasi produttori, la MIC di almeno uno fra $i$ carbapenemi risultava più elevata rispetto al breakpoint clinico di sensibilità; i valori di MIC di ET sono risultati sempre i più elevati, confermando il valore predittivo di tale molecola.

Dieci dei 61 isolati producevano una MBL (8/10 erano VIM-1, 2/10 erano IMP-13).
Tutti gli stipiti produttori di VIM-1 sono risultati intermedi o resistenti ad IP e/o MP $(2 \mathrm{mg} / \mathrm{L} \leq \mathrm{IP} /$ $\mathrm{MP}>8 \mathrm{mg} / \mathrm{L}$ ) con pannelli Microscan; in 2/8 casi sono stati osservati, per il solo IP, valori di MIC inferiori a quelli precedentemente stimati.

Nel caso dei due stipiti di $P$. aeruginosa IMP-13 produttori isolati nel reparto di Terapia Intensiva dell'Ospedale S. Giovanni Rotondo di Foggia, sono stati ottenuti (con pannello NBC45) valori di MIC dei carbapenemi sempre superiori ad 8 $\mathrm{mg} / \mathrm{L}$. Per entrambi gli isolati i valori di MIC per IP e MP risultavano pari a $32 \mathrm{mg} / \mathrm{L}$ mediante microdiluizione in brodo.

Nove isolati di A. baumannii producevano oxacillinasi con spiccata attività carbapenemasica: 4/10

Tabella I. Isolati clinici e relative $\beta L, E S ß L$ e carbapenemasi (CB) prodotte; risultati di identificazione/ performance di rilevamento degli enzimi prodotti mediante il Sistema Microscan (MS).

\begin{tabular}{|c|c|c|c|c|c|c|c|c|c|}
\hline $\begin{array}{l}\text { Isolati } \\
\text { clinici }\end{array}$ & $\begin{array}{c}\mathrm{N} . \\
\text { Isolati }\end{array}$ & $\begin{array}{c}\mathrm{N}^{\circ} \\
\text { ceppi } \beta L \text { positivi }\end{array}$ & $\begin{array}{c}N^{\circ} \text { ceppi } \\
\text { ES } \beta L \\
\text { positivi }\end{array}$ & $\begin{array}{l}N^{\circ} \text { ceppi } \\
\mathrm{CB} \text { positivi }\end{array}$ & $\underset{\text { positivi }}{N^{\circ} \text { ceppi }}$ & Identificazione & $\begin{array}{l}\text { Rilevamento } \\
\text { ES } \beta \mathrm{L}\end{array}$ & $\begin{array}{l}\text { Rilevamento } \\
\text { CB }\end{array}$ & $\begin{array}{l}\text { Rilevamento } \\
E S \beta L+C B\end{array}$ \\
\hline Klebsiella spp. & 21 & $\begin{array}{l}1(K-1)^{*} \\
1(-)\end{array}$ & $\begin{array}{l}4 \text { (CTX-M-15) } \\
1 \text { (SHV-18) }\end{array}$ & $\begin{array}{l}3(\mathrm{VIM}-1) \\
9(\mathrm{KPC} 2 / 3)\end{array}$ & (CTX-M-15 + VIM-I) & $21 / 21$ (100\%) & $5 / 5(100 \%)$ & $12 / 12(100 \%)$ & $.12(0 \%)$ \\
\hline E coli & 17 & $6(-)$ & \begin{tabular}{|l|}
$7($ CTX-M-1/1-14) \\
2 (TEM-101/-52) \\
\end{tabular} & $2(\mathrm{VIM}-1)$ & - & $17 / 17$ (100\%) & $8 / 9(88.8 \%)$ & $2 / 2(100 \%)$ & - \\
\hline P. mirabilis & 15 & $\begin{array}{c}\text { (CMY-16 } \\
+ \text { TEM-1) } \\
3(-) \\
\end{array}$ & $\begin{array}{c}2 \text { (CTX-M-2) } \\
1 \text { (PER-1) } \\
3 \text { (TEM-92) } \\
\end{array}$ & - & - & $15 / 15$ (100\%) & $4 / 6(66.6 \%)$ & - & - \\
\hline Enterobacter spp. & 4 & $\cdot$ & 1 (SHV-12) & $3(\mathrm{VIM})$ & - & $4 / 4(100 \%)$ & $1 / 1(100 \%)$ & $3 / 3(100 \%)$ & $\div$ \\
\hline A. boumonnii & 9 & - & - & $\begin{array}{c}4(\text { (OXA.223) } \\
4(\text { (OXA.58) } \\
1(\text { (sAbaOXA.51) } \\
\end{array}$ & - & 9/9 (100\%) & - & 9/9 (100\%) & - \\
\hline$P$. aeruginosa & 7 & \begin{tabular}{|l|}
1 (mancante di \\
porina OprD2) \\
\end{tabular} & 2 (PER-I) & $\begin{array}{l}2 \text { (IMP-13) } \\
2 \text { (VIM-1/-2) }\end{array}$ & - & $777(100 \%)$ & $2 / 2(100 \%)$ & 4/4 (100\%) & . \\
\hline Totale & 73 & 6 & 23 & 30 & 2 & 73 & $20 / 23(87 \%)$ & $30 / 30(100 \%)$ & $-12(0 \%)$ \\
\hline
\end{tabular}

Tabella 2. Range di MIC degli antibiotici B-lattamici ottenuti con i pannelli Microscan.

\begin{tabular}{|c|c|c|c|c|c|c|c|c|c|}
\hline & & & \multicolumn{7}{|l|}{ FIC (mg/L) } \\
\hline & & & CFX & CAZ & CFT & CPE & IP & MP & ETP \\
\hline \multirow{8}{*}{$\begin{array}{l}\text { I } \\
\text { S } \\
\text { O } \\
\text { L } \\
\text { A } \\
\text { T } \\
\text { I }\end{array}$} & \multirow{4}{*}{ Klebsiella spp. } & $\begin{array}{l}\beta L \\
\text { positivi }\end{array}$ & $\leq 8(* N A)$ & $\leq I(S)$ & $\leq I(S)-8(R)$ & $\leq 1(S)->8(R)$ & $\leq 2(\mathrm{~S})$ & $\leq 2(\mathrm{~S})$ & $\leq 0.5(\mathrm{~S})$ \\
\hline & & $\begin{array}{l}\text { ES } \beta \text { L } \\
\text { positivi }\end{array}$ & $\leq 8-16$ (NA) & $\geq 16(R)$ & $>32(\mathrm{R})$ & $4(\mathrm{l})->8(\mathrm{R})$ & $\leq 1-2(\mathrm{~S})$ & $\leq 0.5-2(\mathrm{~S})$ & $\leq 0.5(\mathrm{~S})$ \\
\hline & & $\begin{array}{l}\text { CB } \\
\text { positivi }\end{array}$ & $\geq 16$ (NA) & $\geq 16(R)$ & $\geq 32$ (R) & $\geq 8(R)$ & $\leq 2(S)-8(R)$ & $\leq 2(\mathrm{~S})->8(\mathrm{R})$ & $>$ I (R) \\
\hline & & $\begin{array}{l}\text { ESBL + } \\
\text { CB } \\
\text { positivi }\end{array}$ & $>8$ (NA) & $>8(R)$ & $>16(R)$ & $>8(\mathrm{R})$ & $4($ (I) & $>1(S-1)-8(I)$ & $\leq 0.5(S)-1(1)$ \\
\hline & \multirow{3}{*}{ E. coli } & $\begin{array}{l}\beta \mathrm{KL} \\
\text { negativi }\end{array}$ & $\leq 8->16(\mathrm{NA})$ & $\leq \mathrm{I}(\mathrm{S})->8(\mathrm{R})$ & $\leq 1(S)-16(R)$ & $\leq 0.5(\mathrm{~S})-4(\mathrm{l})$ & $\leq 2(\mathrm{~S})-8(\mathrm{l})$ & $\leq 0.5 \cdot 2(\mathrm{~S})$ & $\leq 0.5(S)-2(R)$ \\
\hline & & $\begin{array}{l}\text { ES } \beta L \\
\text { positivi }\end{array}$ & $\leq 8-16(N A)$ & $\leq 8(R)-\geq 16$ & $\leq \mathrm{I}(\mathrm{S})->32(\mathrm{R})$ & $\leq 0.5(S)->8(R)$ & $\leq 1(S)-8(\mathrm{l})$ & $\leq 0.5(\mathrm{~S})-8(\mathrm{l})$ & $\leq 0.5(\mathrm{~S})$ \\
\hline & & $\begin{array}{l}\mathrm{CB} \\
\text { positive }\end{array}$ & $8-32$ (NA) & $8(R)-\geq 16$ & $>16(R)$ & $4(\mathrm{I})->8(\mathrm{R})$ & $\leq 1-2(S)$ & $\leq 0.5-2(\mathrm{~S})$ & $\leq 0.5(S)-1(1)$ \\
\hline & \multirow{3}{*}{ P. mirabilis } & $\begin{array}{l}\beta L \\
\text { negativi }\end{array}$ & $\leq 8$ (NA) & $\leq 0.5-1(S)$ & $\leq 0.5-1(\mathrm{~S})$ & $\leq 0.5(\mathrm{~S})-1$ & $\leq 2(\mathrm{~S})$ & $\leq 0.5-2(\mathrm{~S})$ & $\leq 0.5(\mathrm{~S})$ \\
\hline \multirow{8}{*}{$\begin{array}{l}\mathrm{C} \\
\mathrm{L} \\
\mathrm{I} \\
\mathrm{N} \\
\mathrm{I} \\
\mathrm{C} \\
\mathrm{I}\end{array}$} & & $\begin{array}{l}\overline{\beta L} \\
\text { positivi }\end{array}$ & 16 (NA) & $8(R)=\geq 16$ & $\geq 32$ (R) & $\leq 1(S)-8(R)$ & $\leq 2(\mathrm{~S})-4(\mathrm{l})$ & $\leq 2(S)$ & $\leq 0.5(S)$ \\
\hline & & $\begin{array}{l}\text { ES } \beta \mathrm{L} \\
\text { positivi }\end{array}$ & $\geq 8$ (NA) & $\leq 0.5(S)-8(R)$ & $8(R)->32$ & $\geq 8(\mathrm{R})$ & $\leq 2(\mathrm{~S})$ & $\leq 0.5-2(\mathrm{~S})$ & $\leq 0.5(\mathrm{~S})-1(\mathrm{I})$ \\
\hline & A. baumannii & $\begin{array}{l}\text { CB } \\
\text { positivi }\end{array}$ & - & - & - & $>8(\mathrm{R})$ & $4(\mathrm{I})->8(\mathrm{R})$ & $\geq 8(1-R)$ & - \\
\hline & \multirow{3}{*}{ P. aeruginosa } & $\begin{array}{l}\beta \mathrm{L} \\
\text { negativi }\end{array}$ & $>16$ (NA) & $>8(R)$ & - & $>8(R)$ & $>8(R)$ & $8(1)$ & - \\
\hline & & $\begin{array}{l}\text { ES } \beta \text { L } \\
\text { positivi }\end{array}$ & $>16(\mathrm{NA})$ & $>16(R)$ & - & $>8(\mathrm{R})$ & $>8(R)$ & $>8(R)$ & - \\
\hline & & $\begin{array}{l}\mathrm{CB} \\
\text { positivi }\end{array}$ & $>16$ (NA) & $\geq 16(R)$ & - & $>8(R)$ & $>8(R)$ & $>8(\mathrm{R})$ & - \\
\hline & \multirow{2}{*}{ Enterobacter spp. } & $\begin{array}{l}\text { ESßL } \\
\text { positivi }\end{array}$ & $\leq 8(\mathrm{NA})$ & $>8(\mathrm{R})$ & $16(R)$ & $8(R)$ & $\leq 2(S)$ & $\leq 2(S)$ & $\leq 0.5(\mathrm{~S})$ \\
\hline & & $\begin{array}{l}\text { CB } \\
\text { positivi }\end{array}$ & $>32$ (NA) & $>16(R)$ & $>32(R)$ & $\geq 8(\mathrm{R})$ & $\leq 2(S)-\geq 8(I-R)$ & $4(1)-\geq 8(I-R)$ & $>$ I (R) \\
\hline
\end{tabular}

*CFX: cefoxitina; CAZ: ceftazidime; CFT: cefotaxime; CPE: cefepime; IP: imipenem; MP: meropenem; ETP: ertapenem *NA: Non Applicabile 
erano OXA-58-, 4/10 OXA-23- ed 1/10 ISAbaI e bla ${ }_{\text {OXA-51- }}$ positivi. Per tutti questi isolati i valori di MIC per i carbapenemi risultavano sempre intermedi e/o resistenti. Sei dei nove $A$. baumannii produttori di carbapenemasi di tipo OXA hanno mostrato MIC per l'IP superiore ad $8 \mathrm{mg} / \mathrm{L}$; in $3 / 6$ casi anche la MIC del MP era superiore ad $8 \mathrm{mg} / \mathrm{L}$. Tre dei nove $A$. baumannii OXA-58/-51 produttori, testati utilizzando il panello NB40, sono risultati intermedi o resistenti al doripenem ed al meropenem.

\section{DISCUSSIONE}

L'utilizzo di MS ha permesso di rilevare il 91.8\% degli stipiti $\beta$ L-produttori.

I valori di MIC di alcuni carbapenemi (es. IP) sono risultati in qualche caso inferiori di una diluizione rispetto quelli precedentemente determinati (E-test, microdiluizione in brodo); tuttavia, utilizzando i breakpoint EUCAST 2010 per ET, MP and IP, è stato possibile rilevare tutti gli stipiti carbapenemasi produttori.

Il sistema Microscan si è pertanto dimostrato un utile strumento per l'identificazione dei batteri BL, ES $\beta$ L e/o carbapenemasi-produttori.
Il sistema si è inoltre dimostrato particolarmente versatile: l'operatore ha la possibilità non solo di inserire direttamente ulteriori regole esperte, ma anche di variare, in base agli aggiornamenti EUCAST, i criteri di refertazione.

\section{BIBLIOGRAFIA}

1. Kerremans JJ, Verboom P, Stijnene $T$ et al. Rapid identification and antimicrobial susceptibility testing reduce antibiotic use and accelerate pathogen-directed antibiotic use. J Antimicrob Chemother 2008; 61: 428435 .

2. Kollef MH. Broad-spectrum antimicrobials and the treatment of serious bacterial infections: getting it right up front. Clin Infect Dis 2008; 47: S3-S13.

3. Kumar A, Roberts D, Wood KE et al. Duration of hypotension before initiation of effective antimicrobial therapy is the critical determinant of survival in human septic shock. Crit Care Med 2006; 34: 15891596.

4. Harbarth S, Nobre V, Pittet D. Does antibiotic selection impact patient outcome?. Clin Infect Dis 2007; 44: 87-93.

5. Tenover FC, Raney PM., Williams PP et al. Evaluation of the NCCLS Extended-Spectrum BLactamase Confirmation Methods for Escherichia coli with isolates collected during Project ICARE. $J$ Clin Microb 2003; 3142-3146. 